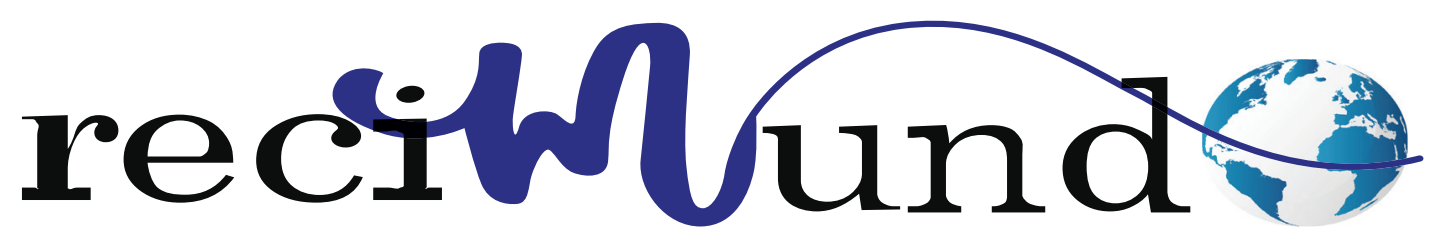

Revista Científica Mundo de la Investigación y el Conocimiento

DOI: 10.26820/recimundo/4.(1).esp.marzo.2020.4-12

URL: http://recimundo.com/index.php/es/article/view/774

EDITORIAL: Saberes del Conocimiento

REVISTA: RECIMUNDO

ISSN: 2588-073X

TIPO DE INVESTIGACióN: Artículo de Revisión

CÓDIGO UNESCO: 5605 Legislación y Leyes Nacionales

PAGINAS: 4-12

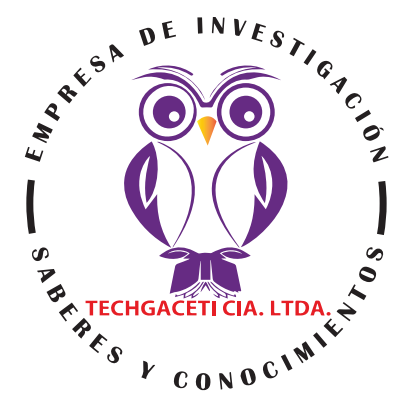

\title{
La interpretación extensiva y la analogía en los delitos de estafa con documentos bancarios
}

Extensive interpretation and analogy in fraud crimes with bank documents Ampla interpretação e analogia em crimes de fraude com documentos bancários

Yohandra Denisse Cevallos López 1; Alba Rosa Pupo Kairuz; Manuel Antonio Calderon Ramírez;;

Dionisio Vitalio Ponce Ruiz ${ }^{4}$

RECIBIDO: 20/11/2019 ACEPTADO: 29/01/2020 PUBLICADO: 05/03/2020

1. Profesional en Formación; Investigador Independiente; Ambato, Ecuador; cevallosyohandra@hotmail.com; https://orcid.org/0000-0002-9156-4762

2. Especialista en Postgrado en Derecho Civil y Familia; Doctora en Ciencias Pedagógicas; Licenciada en Derecho; Investigadora Independiente; Ambato, Ecuador; apupokairuz@gmail.com; ID https://orcid.org/00000002-1719-9250

3. Especialista en Consultoria Jurídico Laboral; Especialista en Derecho Penal y Justicia Indígena; Magister en Derecho Laboral; Abogado de los Tribunales y Juzgandos de la República del Ecuador; Investigador Independiente; Ambato, Ecuador; estjuraristo@hotmail.com; (iD https://orcid.org/0000-0002-3724-9876

4. Master en Nuevas Tecnologías Para la Educación; Doctor en Ciencias Pedagógicas; Ingeniero en Control Automático; Investigador Independiente; Ambato, Ecuador; manzanillo1962@gmail.com; (DD https://orcid. org/0000-0002-5712-4376

\section{CORRESPONDENCIA}

Yohandra Denisse Cevallos López

cevallosyohandra@hotmail.com

\section{Ambato, Ecuador}




\section{RESUMEN}

Desde la óptica de su resultado, la interpretación puede clasificarse en restrictiva, declarativa y extensiva. La aplicación judicial del Derecho dejará de considerarse interpretación cuando se realice al margen del marco establecido por la Ley. La interpretación extensiva de las leyes penales es perfectamente lícita y compatible con el principio de legalidad penal, implica asumir la aplicación más amplia posible de la Ley, también destaca la necesidad de diferenciar entre analogía e interpretación. Es una equivocación: creer que la analogía es una modalidad de interpretación, cuando no es así, la interpretación, incluso cuando se trata de una interpretación de las llamadas extensivas, se hace sobre una norma, la analogía se practica precisamente en ausencia de norma. Para interpretar los derechos fundamentales se pueden tomar en cuenta los siguientes métodos generales de la interpretación jurídica: criterio lógico, sistemático, gramático e histórico. Son documentos bancarios todos aquellos que intervienen en las operaciones realizadas en los bancos, que generan la documentación pertinente en relación al servicio que prestan en áreas: de descuento y de todos los tipos de cuenta corriente, ahorro, etc. La importancia de los Documentos Bancarios es que permiten precisar: gravedad del hecho punible, sofisticación financiera del sospechoso, número de transacciones financieras, valor monetario de las transacciones, número de sospechosos involucrados e identificar y localizar testigos y bienes. Entre los fraudes más comunes que se cometen a diario en el mundo online se encuentran: el robo de identidad, el phishing, el vishing y el smishing. La metodología usada es descriptiva, con un enfoque documental, es decir, revisar fuentes disponibles en la red, como google académico, con contenido oportuno y relevante desde el punto de vista científico que enriquezca el análisis del tema planteado en este artículo.

Palabras clave: Fraude, Estafa, Documentos Bancarios, Vacío Legal, Interpretación Correcta, Cuentas Bancarias, Tarjeta de Crédito, Cheque.

\section{ABSTRACT}

From the perspective of its result, the interpretation can be classified as restrictive, declarative and extensive. The judicial application of the Law will no longer be considered interpretation when it is carried out outside the framework established by the Law. The extensive interpretation of the criminal laws is perfectly lawful and compatible with the principle of criminal legality, implies assuming the widest possible application of the Law It also highlights the need to differentiate between analogy and interpretation. It is a mistake: to believe that analogy is a mode of interpretation, when this is not the case, interpretation, even when it is an interpretation of extensive calls, is made on a norm, the analogy is practiced precisely in the absence of a norm. To interpret fundamental rights, the following general methods of legal interpretation can be taken into account: logical, systematic, grammatical and historical criteria. Banking documents are all those involved in the operations carried out in the banks, which generate the relevant documentation in relation to the service they provide in areas: discount and all types of checking, savings, etc. The importance of the Banking Documents is that they allow us to specify: severity of the punishable act, financial sophistication of the suspect, number of financial transactions, monetary value of the transactions, number of suspects involved and identify and locate witnesses and property. Among the most common frauds that are committed daily in the online world are: identity theft, phishing, vishing and smishing. The methodology used is descriptive, with a documentary approach that is, reviewing sources available on the web, such as google scholar, with timely and scientifically relevant content that enriches the analysis of the topic raised in this article.

Keywords: Fraud, Scam, Bank Documents, Legal Void, Correct Interpretation, Bank Accounts, Credit Card, Check.

\section{RESUMO}

Da perspectiva de seu resultado, a interpretação pode ser classificada como restritiva, declarativa e extensa. A aplicação judicial da lei não será mais considerada interpretação quando for realizada fora do marco estabelecido pela lei. A extensa interpretação das leis penais é perfeitamente lícita e compatível com o princípio da legalidade penal, implica assumir a aplicação mais ampla possível da Lei. Também destaca a necessidade de diferenciar entre analogia e interpretação. É um erro: acreditar que a analogia é um modo de interpretação; quando esse não é o caso, a interpretação, mesmo quando é uma interpretação de chamadas extensas, é feita de acordo com uma norma, a analogia é praticada precisamente na ausência de um norma. Para interpretar os direitos fundamentais, os seguintes métodos gerais de interpretação jurídica podem ser levados em consideração: critérios lógicos, sistemáticos, gramaticais e históricos. Os documentos bancários são todos os envolvidos nas operações realizadas nos bancos, que geram a documentação relevante em relação ao serviço que prestam nas áreas: desconto e todos os tipos de verificação, economia, etc. A importância dos Documentos Bancários é que eles permitem especificar: severidade do ato punível, sofisticação financeira do suspeito, número de transações financeiras, valor monetário das transações, número de suspeitos envolvidos e identificar e localizar testemunhas e propriedades. Entre as fraudes mais comuns cometidas diariamente no mundo on-line estão: roubo de identidade, phishing, vishing e smishing. A metodologia utilizada é descritiva, com uma abordagem documental, ou seja, revisando fontes disponíveis na web, como o google scholar, com conteúdo oportuno e cientificamente relevante que enriquece a análise do tópico levantado neste artigo.

Palavras-chave: Fraude, fraude, documentos bancários, anulação legal, interpretação correta, contas bancárias, cartão de crédito e cheque. 


\section{Introducción}

En la actualidad, es muy sencillo acceder a los extractos bancarios o los movimientos de nuestras tarjetas a través de Internet y las diferentes aplicaciones bancarias. Es importante guardar comprobantes tanto de extractos como de operaciones con tarjetas de crédito. Un buen ejemplo lo tenemos cuando se realiza movimientos en forma de transferencias, o, en el caso de las tarjetas, los comprobantes de movimientos pueden ser vitales ante posibles casos de fraude. No se trata de documentación es que debas guardar durante mucho tiempo, por ejemplo de transferencias que requieran de comprobación o justificación.

La falsificación típicamente tiene lugar cuando un empleado expide un cheque sin la adecuada autorización. Los delincuentes roban un cheque, lo endosan y lo presentan para su pago en una compra local o en el banco en la ventanilla del cajero, utilizando probablemente una falsa identificación personal. La falsificación también puede significar la fabricación completa de un cheque utilizando equipo de impresión de escritorio actualmente disponible, consistente en una computadora personal, scanner, software sofisticado y una impresora láser de alto grado.

Los robo de cheques son prácticas delictivas comunes, sin métodos de detección apropiados, los cheques pasan sin ser descubiertos hasta que él cuenta habiente lo advierte al revisar su estado de cuenta al final del mes. Mientras mayor es la velocidad que crece la tecnología así también responden los fraudes y estafas pero no a esa misma velocidad las Leyes que deben amparar a los ciudadanos.

En esos vacíos muchas veces hay que recurrir en aras de responder con justicia al estafado a la interpretación de determinada Ley o normativa existente, lo cual no siempre se logra, es por ello que conviene estar atentos a estas prácticas delincuenciales a fin de evitarlas, en caso de ocurrir saber diferenciar cuando corresponde una interpretación extensiva o analogía de la Ley como se explicará brevemente en el siguiente artículo.

\section{Metodología}

Esta investigación está dirigida al estudio de "La interpretación extensiva y la analogía en los delitos de estafa con documentos bancarios". Para realizarlo se usó una metodología tipo descriptiva, con un enfoque documental, es decir, revisar fuentes disponibles en la red, como google académico, con contenido oportuno y relevante desde el punto de vista científico para dar respuesta a lo tratado en el presente artículo y que sirvan de inspiración para realizar otros proyectos. Las mismas pueden ser consultadas al final, en la bibliografía.

\section{Resultados}

\section{Interpretación extensiva y analogía}

Desde la óptica de su resultado, la interpretación puede clasificarse en restrictiva, declarativa y extensiva. La aplicación judicial del Derecho dejará de considerarse interpretación cuando se realice al margen del marco establecido por la Ley, es decir, cuando dicha aplicación judicial no esté cubierta por el sentido literal posible de un precepto penal, se producirá entonces, una inadmisible e ilegal analogía fundamentadora de la pena (Ribas, 2014). De las tres clases de interpretación referidas la más próxima a la analogía y la más problemática, es la denominada interpretación extensiva, que implica atribuir a un término legal un elevado número, sino el máximo, de acepciones permitidas por su tenor literal, no puede ser rebasado en ningún caso.

Aunque suele identificarse la interpretación extensiva como la comprensión más amplia posible del texto legal cabría afirmar que 


\section{LA INTERPRETACIÓN EXTENSIVA Y LA ANALOGÍA EN LOS DELITOS DE ESTAFA CON DOCUMENTOS BANCARIOS}

los tipos legales tendrían un contenido mínimo y un contenido máximo, dando lugar a diversas clases de interpretaciones y entre ellas a la interpretación extensiva que sería la que diera al texto legal el contenido máximo de casos. Entendida de esta forma, la interpretación extensiva puede considerarse, respetuosa con el principio de legalidad y aceptable. Por el contrario, si la interpretación extiende el sentido de la norma más allá de las acepciones posibles del término legal (su sentido literal), está permitiendo su aplicación a supuestos no contenidos en el precepto y, por tanto, incurriendo en analogía prohibida.

La interpretación extensiva de las leyes penales es perfectamente lícita y compatible con el principio de legalidad penal, ya que implica asumir la aplicación más amplia posible de la Ley, también destaca la necesidad de diferenciar entre analogía e interpretación. Según Quintero Olivares es común incluir la referencia a la analogía al hablar de la interpretación, lo cual obliga a alertar de una posible equivocación: la de creer que la analogía es una modalidad de interpretación, cuando no es así, la interpretación, incluso cuando se trata de una interpretación de las llamadas extensivas, se hace sobre una norma (Ribas, 2014). La analogía se practica precisamente en ausencia de norma.

En ocasiones se pretende calificar de simple "interpretación extensiva" lo que es pura analogía a causa de que el intérprete se ha alejado tanto de la norma analizada, lo que pretende sostener es algo que ya no puede caber en esa norma. Probablemente, la analogía deba diferenciarse de la interpretación extensiva. Aun cuando ambos conceptos son sumamente debatidos, no se encuentran claramente deslindados. La interpretación extensiva es aquella que supone una intelección amplia de la norma, dando cobertura dentro de ella al máximo número de supuestos de hecho posibles, pero siempre dentro del marco trazado por su tenor literal, de modo que si se desborda éste se incide en la analogía. Implicaría, en definitiva, seleccionar entre las diversas opciones aplicativas aquella que supone dar una mayor cabida a la norma.

Los casos que se suponen comprendidos en el supuesto de hecho de la norma de acuerdo con el método teleológico resultan impracticable para establecer el límite máximo de la interpretación permitida frente a la analogía prohibida, por lo que la solución debe ser la exclusión de toda interpretación extensiva en el derecho penal. la extensión de la ley penal por encima de la interpretación que permita fijar un número mínimo de casos comprendidos en su texto es violatoria de la función de garantía de la ley penal, de modo que no es el sentido posible del texto lo que dará el límite de la interpretación sino el sentido más restringido del texto.

Para interpretar los derechos fundamentales se pueden tomar en cuenta los siguientes métodos generales de la interpretación jurídica (Carbonell, 2004):

- Criterio lógico, según el cual hay que entender las normas de derecho fundamental como si fuesen consistentes con las demás normas constitucionales; el intérprete no puede representar el texto constitucional como una serie de enunciados ilógicos, sino que debe proceder de tal forma que se advierta una cierta coherencia normativa. Esto no evita, ni el intérprete puede tampoco contribuir a disimularlo, que entre las normas constitucionales que contemplan derechos fundamentales pueda haber contradicciones o tensiones, en cuyo caso se tendrán que tomar en cuenta criterios hermenéuticos adicionales, como lo son la ponderación o la proporcionalidad.

- Criterio sistemático, según el cual hay que considerar a la Constitución como una unidad, de manera que el intérprete debe enlazar las normas de derecho

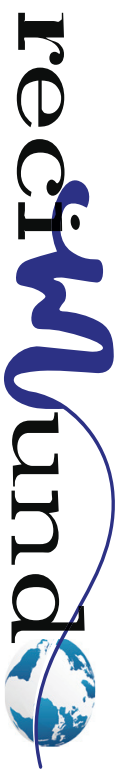


fundamentales entre sí, descubriendo su sentido y alcances en relación con el sentido y alcances del resto del texto constitucional. El derecho fundamental debe ser contextualizado en el conjunto de la Constitución para lograr su adecuada interpretación.

- Criterio gramatical o filológico, según el cual debe atenderse al significado lingüístico contenido en las normas de derechos fundamentales. La interpretación lingüística, sin embargo, no se debe limitar al significado que nos ofrecen los diccionarios, sino que comprende también el significado que la cultura y la tradición jurídicas le dan a cierto término; igualmente, el significado lingüístico puede ser conocido a través de lo que se haya establecido en sentencias que constituyan precedentes obligatorios, en sentencias de tribunales de otros países y por el resto de órganos encargados de aplicar el derecho, incluyendo, desde luego, los significados lingüísticos individualizados por los teóricos de los derechos fundamentales.

- Criterio histórico, según el cual hay que intentar precisar el sentido que a una determinada norma de derecho fundamental le dio el poder constituyente o el poder reformador de la Constitución. La reconstrucción de la voluntad constituyente se puede realizar por medio de los debates parlamentarios, de las exposiciones de motivos que fundamentaron determinadas iniciativas de reforma, o por la legislación histórica sobre la materia (Rodriguez, 2013).

\section{Estafa}

Cometen estafa los que, con ánimo de lucro, utilizan el engaño para producir error en otro, induciéndolo a realizar un acto de disposición en perjuicio propio o ajeno. El delito de estafa se encuentra tipificado en el art. 248 del Código penal de Ecuador. También se consideran autores del delito de estafa (Castillo, 2019):
- Los que, con ánimo de lucro y valiéndose de alguna manipulación informática o artificio semejante, consigan una transferencia no consentida de cualquier activo patrimonial en perjuicio de otro.

- Los que fabricaren, introdujeren, poseyeren o facilitaren programas informáticos específicamente destinados la comisión de las estafas previstas en lo señalado en este artículo 248.

La Sentencia del Tribunal Supremo de 26.12.2014 dice (Castillo, 2019): los elementos que estructuran el delito de estafa, a tenor de las pautas que marcan la doctrina y la jurisprudencia, son los siguientes:

a. La utilización de un engaño precedente, bastante y causante, por parte del autor del delito, para generar un riesgo no permitido para el bien jurídico (primer juicio de imputación objetiva); esta suficiencia, idoneidad o adecuación del engaño ha de establecerse con arreglo a un baremo mixto objetivo-subjetivo, en el que se pondere tanto el nivel de perspicacia o intelección del ciudadano medio como las circunstancias específicas que individualizan la capacidad del sujeto pasivo en el caso concreto.

b. El engaño ha de desencadenar el error del sujeto pasivo de la acción.

c. Debe darse también un acto de disposición patrimonial del sujeto pasivo, debido precisamente al error, en beneficio del autor de la defraudación o de un tercero, desplazamiento que no se habría producido de resultar conocida la naturaleza real de la operación.

d. La conducta engañosa ha de ser ejecutada con dolo y ánimo de lucro.

e. De ella tiene que derivarse un perjuicio para la víctima, perjuicio que ha de aparecer vinculado causalmente a la acción engañosa (nexo causal o naturalístico) y materializarse en el mismo el riesgo ilícito que para el patrimonio de la víctima supone la acción engañosa del sujeto activo (relación de riesgo o segundo juicio de imputación objetiva). 


\section{LA INTERPRETACIÓN EXTENSIVA Y LA ANALOGÍA EN LOS DELITOS DE ESTAFA CON DOCUMENTOS BANCARIOS}

Son documentos bancarios todos aquellos que intervienen en las operaciones realizadas en los bancos, que generan la documentación pertinente en relación al servicio que prestan en áreas:

a. De descuento: avalan operaciones de descuento de documentos mercantiles negociables como la letra de cambio y el pagare.

b. De todos los tipos de cuenta corriente, ahorro, etc. Es un contrato entre el banco (custodio) y el cliente (agente económico excedentario) en el cual el banco se compromete a cumplir con órdenes de pago a terceros hasta el límite de dinero depositado en dicha cuenta.

La importancia de los Documentos Bancarios es que permiten precisar (Infante, 2008):

- Gravedad del hecho punible.

- Sofisticación financiera del sospechoso.

- Número de transacciones financieras.

- Valor monetario de las transacciones.

- Número de sospechosos involucrados.

- Identificar y localizar testigos y bienes.

Dentro de las prácticas irregulares más usualmente utilizadas por las administraciones bancarias en la crisis financiera de 1998 en Ecuador, podemos encontrar (Raza, Mayo 2008):

1. Políticas de mercado excesivamente agresivas, que con el propósito de captar el mayor número de clientes, descuidan las seguridades mínimas que debían guardar las instituciones financieras, tanto en la aceptación de captaciones, como en la colocación de activos financieros y que desencadenaron en incrementos substanciales de los niveles de riesgo a que las instituciones se expusieron. Esta práctica en otros países es conocida como oferta engañosa de productos bancarios y en el Ecuador se ha manifestado en difusiones publicitarias que inducía al error de los depositantes, con falsas expectativas de altas tasas de interés, de pago de rendimientos diarios, de solvencia y consistencia financieras de las instituciones, de premios, loterías, viajes, sueldos de por vida, etc.

2. Concentración del crédito en determinados segmentos de la economía, lo que hace depender gran parte de la cartera y su recuperación, de las condiciones en que dicho segmento se encuentre.

3. Concentración de créditos vinculados en condiciones preferenciales o desproporcionadas en relación con su patrimonio o con el de la institución financiera, en muchos casos con infracción a los límites máximos previstos por la Ley vigente.

4. Ejecución de actos ilícitos, de aquellos prohibidos por la Ley, tanto para incrementar las utilidades como para ocultar la real posición financiera de la institución, producto de otras prácticas irregulares como los créditos vinculados, así como para inyectarle recursos ficticios o provenientes de la legitimación ilegal de capitales, tanto a la institución, como a las demás empresas del grupo financiero.

5. Inconsistente calificación de la cartera de créditos, a través de sus Comités de Crédito calificaban a sus activos como clase A, (poco riesgo) cuando se presentan los problemas de recuperación de cartera, la realidad es completamente distinta.

6. Baja capitalización de las instituciones, manifestada en que el capital y reservas de todo el sistema bancario, representa solo la séptima parte de los activos, es decir del total de créditos otorgados, era solo una pequeña fracción de los fondos movilizados (Carpio, Octubre 2016).

7. Bajas provisiones destinadas por los bancos para eventuales pérdidas, que demuestra paradójicamente que mientras el cobro de los créditos se hacía más difícil, las provisiones disminuían, es decir que el colchón para enfrentar las pérdidas es cada vez menor.

8. Concesión de créditos de liquidez por parte del Banco Central del Ecuador, esta es una práctica irregular en la medida que

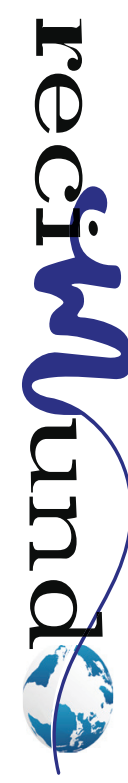


los bancos, conocedores de esta posibilidad acuden a créditos de liquidez, créditos subordinados y programas de estabilización al amparo de lo previsto en la Ley de Régimen Monetario y Banco del Estado, para cubrir sus deficiencias patrimoniales y para sostener sus niveles de liquidez.

\section{Modalidades de fraude}

- Con cheques: comprende la falsificación, adulteración (cambian datos del cheque como borrar el número de cuenta) o el uso de cheques en blanco obtenidos ilícitamente. La falsificación de la firma en documento y alteración de caracteres magnéticos también constituyen delitos de este tipo (Ramírez, 2008).

- Con tarjeta de crédito: clonación de tarjeta, compras en caliente o con tarjetas recientemente robadas que no tienen ningún tipo de denuncio o bloqueo, falsificación integral de la tarjeta, alteración de la banda magnética, aumento fraudulento de cupo, suplantación de la razón social, cambio de tarjeta, cédula falsa, fuga de información, lavado de dinero mediante el uso de estas tarjetas, etc. (Gómez, 2012).

\section{Fraudes electrónicos}

Entre los fraudes más comunes que se cometen a diario en el mundo online se encuentran: el robo de identidad, el phishing, el vishing y el smishing (Oxman, Diciembre 2013):

Robo de identidad: es la usurpación o la apropiación de su identidad personal y tarjetas de crédito, sin el permiso del individuo, utilizan este robo para compras online, alquilar propiedades o abrir otras cuentas a sus nombres.

Phishing: viene de la palabra inglesa, "fishing" que significa pesca (Flores, 2014) y es el robo de datos personales, que se da por medio de hackers que envían correos fraudulentos o enlaces a correos electrónicos sospechosos, con el fin de obtener contraseñas de cuentas bancarias y números de tarjetas de crédito.

Vishing: consiste en robar identidad por voz IP que recrea una llamada de una entidad financiera o un banco, por ejemplo, engañando a un individuo para que le brinde información confidencial como contraseñas y número de cédula, es un phishing que se hace por teléfono.

Smishing: este fraude ocurre por medio de mensajes de texto y sencillamente invitan al usuario a hacer clics en enlaces fraudulentos para estafarlos, robando sus contraseñas de banco y de correos electrónicos, a veces se valen también de formularios que las personas deben de llenar, haciéndoles creer que pueden participar para un premio o aprovechar una oferta de una tienda virtual.

\section{Delitos Informáticos tipificados en la Le- gislación Ecuatoriana}

La Ley de Comercio Electrónico, Firmas Electrónicas y Mensajes de Datos más conocida como Ley 67, publicada en el R.O. / Sup.557 del 17 de Abril del 2002, tuvo un avance muy importante al incluir figuras penales que hagan punibles los ilícitos informáticos, con lo cual, junto al Código Penal, integran normas creadas para la Sociedad de la Información. Dentro de estas normas promulgadas en la Ley 67, posteriormente incluidas al Código Penal, constan los siguientes ilícitos informáticos (Colón, Junio 2014).

Art. 57 LCEFEMD: Infracciones informáticas. Se considerarán infracciones informáticas, las de carácter administrativo y las que se tipifican, mediante reformas al Código Penal, en la presente ley.

Art. 58 LCEFEMD, Conc. Art. 202.1 CP: Contra la Información Protegida. El que em- 


\section{LA INTERPRETACIÓN EXTENSIVA Y LA ANALOGÍA EN LOS DELITOS DE ESTAFA CON DOCUMENTOS BANCARIOS}

pleando cualquier medio electrónico, informático o afín, violentare claves o sistemas de seguridad, para acceder $u$ obtener información protegida, contenida en sistemas de información; para vulnerar el secreto, confidencialidad y reserva, o simplemente vulnerar la seguridad, será reprimido con prisión de seis meses a un año y multa de quinientos a mil dólares de los Estados Unidos de Norteamérica. Si la información obtenida se refiere a seguridad nacional, o a secretos comerciales o industriales, la pena será de uno a tres años de prisión y multa de mil a mil quinientos dólares de los Estados Unidos de Norteamérica. La divulgación o la utilización fraudulenta de la información protegida, así como de los secretos comerciales o industriales, serán sancionadas con pena de reclusión menor ordinaria de tres a seis años y multa de dos mil a diez mil dólares de los Estados Unidos de Norteamérica. Si la divulgación o la utilización fraudulenta se realizan por parte de la persona o personas encargadas de la custodia o utilización legítima de la información, éstas serán sancionadas con pena de reclusión menor de seis a nueve años y multa de dos mil a diez mil dólares de los Estados Unidos de Norteamérica.

Art.58 últ. inc LCEFEMD, Conc. Art. 202.2 CP: Obtención y utilización no autorizada de información. La persona o personas que obtuvieren información sobre datos personales para después cederla, publicarla, utilizarla o transferirla a cualquier título, sin la autorización de su titular o titulares, serán sancionadas con pena de prisión de dos meses a dos años y multa de mil a dos mil dólares de los Estados Unidos de Norteamérica.

Art.59 LCEFEMD, Conc. Art. 262 CP: Destrucción Maliciosa de Documentos. Serán reprimidos con tres a seis años de reclusión menor, todo empleado público y toda persona encargada de un servicio público, que hubiere maliciosa y fraudulentamente, destruido o suprimido documentos, títulos, programas, datos, bases de datos, información o cualquier mensaje de datos contenido en un sistema de información o red electrónica, de que fueren depositarios, en su calidad de tales, o que les hubieren sido encomendados sin razón de su cargo.

Art.60 LCEFEMD, Conc. Art. 353.1 CP: Falsificación electrónica. Son reos de falsificación electrónica la persona o personas que con ánimo de lucro o bien para causar un perjuicio a un tercero, utilizando cualquier medio; alteren o modifiquen mensajes de datos, o la información incluida en éstos, que se encuentre contenida en cualquier soporte material, sistema de información o telemático, ya sea: Alterando un mensaje de datos en alguno de sus elementos o requisitos de carácter formal o esencial; simulando un mensaje de datos en todo o en parte, de manera que induzca a error sobre su autenticidad.

\section{Conclusiones}

La aplicación judicial del Derecho dejará de considerarse interpretación cuando se realice al margen del marco establecido por la Ley, es decir, cuando dicha aplicación judicial no esté cubierta por el sentido literal posible de un precepto penal, se producirá entonces, una inadmisible e ilegal analogía fundamentadora de la pena. Por el contrario, si la interpretación extiende el sentido de la norma más allá de las acepciones posibles del término legal (su sentido literal), está permitiendo su aplicación a supuestos no contenidos en el precepto y, por tanto, incurriendo en analogía prohibida.

Cometen estafa los que, con ánimo de lucro, utilizan el engaño para producir error en otro, induciéndolo a realizar un acto de disposición en perjuicio propio o ajeno. El delito de estafa se encuentra tipificado en el art. 248 del Código penal de Ecuador. También se consideran autores del delito de estafa: los que, con ánimo de lucro y valiéndose de alguna manipulación informática o artificio

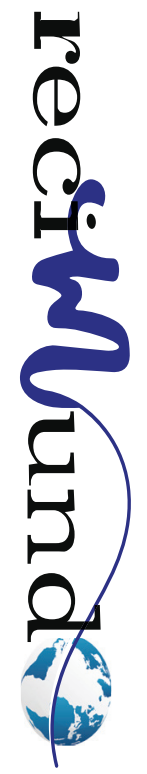


semejante, consigan una transferencia no consentida de cualquier activo patrimonial en perjuicio de otro. Los que fabricaren, introdujeren, poseyeren o facilitaren programas informáticos específicamente destinados la comisión de las estafas previstas en lo señalado en este artículo 248.

Los delitos más comunes como fraudes son: con cheques la falsificación, adulteración (cambian datos del cheque como borrar el número de cuenta) o el uso de cheques en blanco obtenidos ilícitamente. La falsificación de la firma en documento y alteración de caracteres magnéticos también constituyen delitos de este tipo. Con tarjeta de crédito: clonación de tarjeta, compras en caliente o con tarjetas recientemente robadas que no tienen ningún tipo de denuncio o bloqueo, falsificación integral de la tarjeta, alteración de la banda magnética, aumento fraudulento de cupo, suplantación de la razón social, cambio de tarjeta, cédula falsa, fuga de información, lavado de dinero mediante el uso de estas tarjetas, etc. Entre los fraudes más comunes que se cometen a diario en el mundo online se encuentran: el robo de identidad, el phishing, el vishing y el smishing.

\section{Bibliografía}

Carbonell, M. (2004). La Interpretación de los Derechos Fundamentales. Revista lus et Praxis Año 10 No1, 409 - 417 https://scielo.conicyt.cl.

Carpio, J. D. (Octubre 2016). Sobre la necesaria interpretación y aplicación restrictiva del delito de blanqueo de capitales. INDRET Revista para el análisis del Derecho., 1 - 44 WWW. INDRET.COM.
Castillo, I. (04 de Noviembre de 2019). Los elementos del delito de estafa. Obtenido de Mundojuridico. info: https://www.mundojuridico.info

Colón, E. (Junio 2014). Cómo responder a un delito informático. Revista Ciencia UNEMI No 11, 43 - 50 http://181.188.214.100.

Flores, F. (2014). Respuesta penal al denominado robo de identidad en las conductas de phishing bancario. Revista de Estudios Penales y Criminológicos, http://www.usc.es.

Gómez, J. (2012). Los delitos de estafa en el tráfico inmobiliario. Granada: Editorial COMARES S.L.

Infante, R. (26 de Noviembre de 2008). Docuemntos Comerciales Bancarios. Obtenido de Gestiopolis: https://www.gestiopolis.com

Oxman, N. (Diciembre 2013). Estafas informáticas a través de Internet: acerca de la imputación penal del "phishing" y el "pharming. Revista de Derecho de la Pontificia Universidad Católica de Valparaíso No.41, https://scielo.conicyt.cl.

Ramírez, N. (2008). El fraude en la actividad bancaria. El Cuaderno - Escuela de Ciencias Estratégicas Vol 2 n 4, 279 - 296 www. dialnet.unirioja.es.

Raza, S. (Mayo 2008). El peculado bancario en la crisis financiera de 1998. Serie Magister Volumen 81, http://www.cenlibrosecuador.org.

Ribas, E. (2014). INTERPRETACIÓN EXTENSIVA Y ANALOGÍA EN EL DERECHO PENAL. UNED. Revista de Derecho Penal y Criminología, Volumen 3, No12, 111 - 164 http://e-spacio.uned.es.

Rodriguez, J. (2013). EL CRITERIO HISTÓRICO EN LA INTERPRETACIÓN JURÍDICA. Revista Dereito Vol.22, 599 - 632 http://www.usc.es.

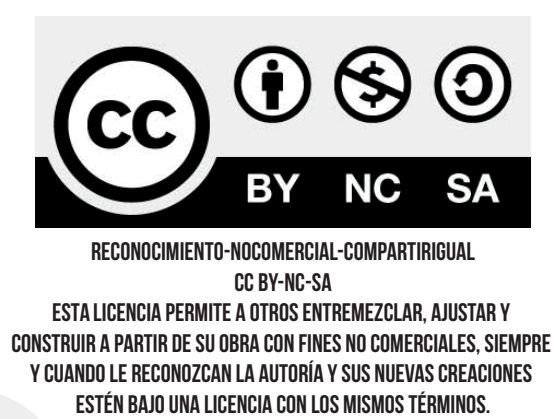

\section{CITAR ESTE ARTICULO:}

Cevallos López, Y., Pupo Kairuz, A., Calderon Ramírez, M., \& Ponce Ruiz, D. (2020). La interpretación extensiva y la analogía en los delitos de estafa con documentos bancarios. RECIMUNDO, 4(1(Esp)), 4-12. doi:10.26820/recimundo/4.(1).esp.marzo.2020.4-12 